\title{
Parameter Identification for Respiratory Dynamics by Personalized Simulation and Experiment*
}

\author{
Shigeo WADA**, Yasuyuki SEGUCHI***, Masao TANAKA****, \\ Mitsumasa MATSUDA*****, Taiji ADACHI ${ }^{* * * * * *}$ \\ and Motoharu FUJIGAKI******
}

\begin{abstract}
Gas flow in the airway and lung deformation due to tissue elasticity and surface tension are the main characteristics of respiratory dynamics. In this study, a simulation with the discretized model as a distributed parameter system is used extensively to identify some of the model parameters which could not be measured directly, and to estimate the real situation of lung respiration. With the use of 5 excised lungs from Japanese white rabbits, the pleural pressure vs lung volume change $\left(P_{p l}-\Delta V\right)$ relations are observed in situ under oscillating pleural pressure. The elastic properties of the lung tissue are measured under biaxial tension. The unknown parameters, such as that for the surface tension in the alveoli, are identified so that the $P_{p l}-\Delta V$ relations calculated by the simulation coincide with the experimental ones for an individual lung. The simulation studies with identified parameters demonstrate potential for estimation of the actual phenomena inside of an excised lung.
\end{abstract}

Key Words: Biomechanics, Simulation, Lung, Dynamics, Identification, Breathing, Respiration, Material Testing, Surface Tension

\section{Introduction}

The lung respiratory system is a large-scale mechanical system having complexity which stems from the structural hierarchy, the geometrical and material nonlinearities, the multifield interactions and so on. As the basic function of a lung, alveolar gas is exchanged with the external fresh air. The process is primarily driven by the mechanical behavior of the lung. In the clinical field, respiratory function is conventionally evaluated from the physiological view.

* Received 5th November, 1991. Paper No. 90-1264 A

** Faculty of Science and Technology, Ryukoku University, Seta, Otsu 520-21, Japan

*** Formerly, Faculty of Engineering Science, Osaka University, 1-1 Machikaneyama, Toyonaka, Osaka 560, Japan

**** Faculty of Engineering Science, Osaka University, 1-1 Machikaneyama, Toyonaka, Osaka 560, Japan

***** Graduate School of Science and Technology, Kobe University, 1-1, Rokkodai, Nada, Kobe 657, Japan

***** Division for Research of Engineering Science, Graduate School of Osaka University, 1-1 Machi. kaneyama, Toyonaka, Osaka 560, Japan point. However, it is important to approach the study of lung respiration from a mechanical viewpoint.

Much effort has been devoted to several mechanical aspects of breathing dynamics. Research typically involves the continuum models for lung deformation ${ }^{(1) \sim(3)}$, airway morphology $y^{(4),(5)}$, gas dynamics in the airway ${ }^{(6),(7)}$, the surface tension properties of the gas-tissue interface at alveoli ${ }^{(8),(9)}$, and so on. Some mathematical models ${ }^{(10),(11)}$ have been proposed for the deformation of the excised lung, on the basis of experimental observations of the pressure -volume relation ${ }^{(12)}$ and indentation properties ${ }^{(13)}$.

The authors have also developed a mathematical model which represents the actual structure of lung respiration as much as possible. The concept of analysis by synthesis was employed in order to allow the model to be used for the quantitative understanding of a respiratory phenomena by means of simulation $^{(14) \sim(20)}$. Since direct observations of the mechanical properties in vivo and in vitro are limited to those such as the tissue elasticity and the pressurevolume relation of the excised lung, simulation becomes a key element in the identification of the model parameters. This enables us to unify the ele- 
ment-level and the system-level observations in the limited experiments, and to identify and visualize the internal phenomena which could not be directly observed.

In this study, experiments are first conducted to obtain the pressure-volume relations and the tissue elasticity of the lungs excised from Japanese white rabbits. Some model parameters are identified for each lung specimen, and the simulation model of lung respiration is established individually. Then, the study focuses on the personalized simulation of lung respiration in order to investigate quantitatively the hidden phenomena inside of the lung, such as surface tension at the alveoli.

\section{Breathing Model}

The simulation model of breathing reported in the previous articles ${ }^{(14) \sim(17)}$ is briefly described in this section. Two stress factors make the macroscopic stress of the lung parenchyma: the elastic stress $\sigma_{e}$ due to tissue elasticity and the stress $\sigma_{s}$ due to surface tension at the gas-tissue interface at alveoli ${ }^{(1)}$, that is,

$$
\sigma=\sigma_{e}+\sigma_{s}
$$

The elastic stress $\sigma_{e}$ is derived from the pseudostrain energy function proposed by Fung ${ }^{(1)}$, as shown in the Appendix, and is written as the function of the stretch ratio $\lambda$ of the lung tissue when the parenchyma is idealized as the bulk of cubic alveoli with edge length $\Delta$. In this context, the stress $\sigma_{s}$ is related to the surface tension $\gamma$ as

$$
\sigma_{s}=\frac{4 \gamma}{\Delta \lambda}
$$

where the hysteresis of the surface tension against the cyclic change of the surface area is represented by the rate-type model

$$
\frac{d r}{d t}=\left\{\begin{array}{l}
a\left(\gamma_{u}-\gamma\right) d \Lambda / d t, d \Lambda / d t \geqq 0 \\
a\left(\gamma-\gamma_{\iota}\right) d \Lambda / d t, d \Lambda / d t<0
\end{array}\right.
$$

proposed in previous reports ${ }^{(16),(17)}$. The surface area ratio $\Lambda$ is equal to the square of the stretch ratio $\lambda$ under uniform inflation of the cubic alveoli. The upper and lower bounds $\gamma_{u}$ and $\gamma_{l}$ of the surface tension, and the coefficient $a$ in Eq. ( 3 ) characterize the features of hysteresis, such as the slope of the loop and the hysteresis area.

For the breathing dynamics, the equations for gas continuity and gas motion are provided for each generation of the airway. The sets of these two equations at the generations from the mouth to the alveoli are connected by taking into account the pressure drop and the gas continuity at the branching points of the airway, and this yields the system equation for the gas flow of the breathing dynamics ${ }^{(17)}$. The loss of pressure head $h_{i}$ at the branch between the $i$ th and $i+1$ th generations is written as a quadratic function of the gas velocity $v_{i}$ in the $i$ th generation,

$$
h_{i}=\zeta_{i} v_{i}^{2} / 2
$$

where $\zeta_{i}$ is the pressure loss coefficient. The alveolar pressure, a boundary condition of the gas-flow submodel, is calculated using the equilibrium condition at the interface of the lung parenchyma and the pleural space

$$
P_{a l}=P_{p l}+\sigma
$$

where the pleural pressure $P_{p l}$ functions as the boundary condition of the overall model, which drives the breathing.

The total lung capacity $V$ is evaluated as the sum of the alveolar space $V_{a l}$ and the volume of conducting airway $V_{c n}$, and is written as

$$
V=V_{a l}+V_{c n} \text {. }
$$

The total volume of alveoli $V_{a l}$ is expressed as

$$
V_{a l}=V_{0} \lambda^{3}
$$

under uniform inflation of the lung, whose volume of alveoli is $V_{0}$ in the natural state, free from elastic stress. The time derivative of the total lung capacity gives the gas flow through the mouth,

$$
d V / d t=A_{0} v_{0}(0, t)
$$

where $A_{0}$ and $v_{0}$ are the cross-sectional area of the airway and the gas velocity at the 0 th generation, respectively.

The partial differential equations for the gas-flow submodel are discretized by the finite-element method, based on the adjoint variational principle ${ }^{(17)}$. With the use of the step-by-step method combined with the conventional iterative technique, the obtained ordinary differential equations are solved simultaneously under the boundary conditions related to the lung deformation, and then the pleural pressure $P_{p l}$ and the volume change of the lung $\Delta V$ are related to each other through the simulation.

\section{Experimental Observation with Excised Lung}

The mechanical properties are examined for the excised lung from the viewpoint of personalized modeling. The pressure-volume relations of the lung are examined as system-level characteristics of the breathing dynamics and the elasticity of the lung tissue is tested as an element-level property.

\section{1 Pressure-volume relations of entire lung}

In the lungs excised from Japanese white rabbits with a weight of about $3 \mathrm{~kg}$, the relations between the pleural pressure and the volume change of the lung $\left(P_{p l}-\Delta V\right.$ relations) are observed in situ. The lung specimen is hung in the pleural chamber at the trachea shown in Fig 1. The upper trachea is opened to the atmosphere through the flowmeter (PNEUMOTACHOGRAPH Model i/a 7317 from Dynascience Co. combined with pressure transducer Model DP 45 from Validyne Co.). The rubber diaphragm placed at the 
bottom of the pleural chamber is moved by the electro -hydro servo testing machine (Model PSA-01 from Tokyo Koki-Schenk), and it oscillates the internal pressure of the chamber, which imitates the pleural pressure. This fluctuation is detected by the pressure transducer (Model SCX 01 DN from Sen-Sym Co.). The velocity of gas flow at the upper trachea and the pressure in the pleural chamber are recorded simultaneously under steady oscillation of the pleural pressure after several cycles of preconditioning. The sampling interval is $5.4 \mathrm{msec}$. The volume change of the lung is calculated by the time integration of the volumetric flow. The recorded data were smoothed by means of the moving average scheme with a thirdorder polynomial fitting over the sequence of 11 data for a $54 \mathrm{msec}$ period in a rolling manner.

Five rabbit lungs (specimens $I-\mathrm{V}$ ) are examined under several conditions of pleural pressure oscillation. The upper bound of pleural pressure is fixed at $-0.49 \mathrm{kPa}$, and the pleural pressure oscillates with a peak-to-peak amplitude of (A) $0.49 \mathrm{kPa}$, (B) 0.98 $\mathrm{kPa}$ and $(\mathrm{C}) 1.47 \mathrm{kPa}$ at frequencies of $0.2 \mathrm{~Hz}, 0.5 \mathrm{~Hz}$, $1 \mathrm{~Hz}, 2 \mathrm{~Hz}, 5 \mathrm{~Hz}$ and $10 \mathrm{~Hz}$.

In order to characterize the hysteresis of the $P_{p l}$ $-\Delta V$ relations shown in Fig. 2, the tidal volume $\bar{V}$ and the area of hysteresis $H$ are calculated from the $P_{p l^{-}}$ $\Delta V$ relations. Table 1 shows the obtained results in the cases of the frequencies of $0.2 \mathrm{~Hz}$ and $5 \mathrm{~Hz}$.

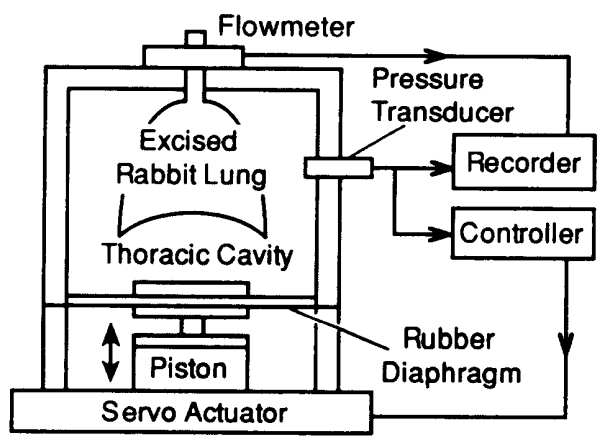

Fig. 1 Experimental apparatus for measurement of $P_{p t^{-}}$ $\Delta V$ relation

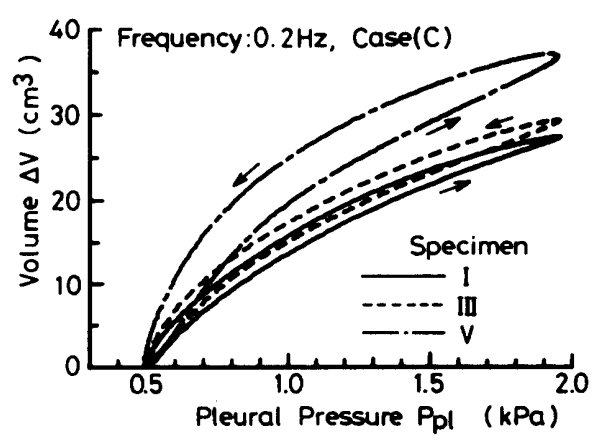

Fig. 2 Observed $P_{p l}-\Delta V$ relations

\subsection{Elastic properties of lung tissue}

Following the $P_{p l}-\Delta V$ test, the biaxial tension test is conducted to investigate the elastic properties of each lung tissue. Excised lung is frozen at $-40^{\circ} \mathrm{C}$, and a test piece with dimensions of about $20 \times 20 \times 3$ $\mathrm{mm}$ is extracted from the center of the underlobe by means of a microtome cutter. The test piece is then thawed at room temperature, and is stained by india ink in the central region with 25 dot marks.

With reference to the experimental setup of Vawter et al. ${ }^{(21)}$, the apparatus shown in Fig. 3 is designed and used for the biaxial tension test. The test piece is immersed in a saline bath and kept free from influence of the surfactant and the surface tension. Uniform tensile force is loaded to the test piece at each edge through 6 threads. The tension in the $x$ direction is equalized to that in the $y$ direction, which is measured by a force transducer (Model DFG- $0.2 \mathrm{~K}$ by Simpo Industry $\mathrm{Co}_{0}$.). The movement of the dot marks on the test piece is monitored by a CCD camera system through the window at the bottom of the saline bath, and the tissue deformation is evaluated by means of the image processing technique. Quasi-static tension is applied to the test piece in a cyclic manner until a steady response is realized. The stretch ratio

Table 1 Mechanical properties of excised lungs $\left(\begin{array}{l}\text { Case (A) : } P_{p l}=-0.49 \sim-0.98 \mathrm{kPa} \\ \text { Case (B) : } P_{p l}=-0.49 \sim-1.47 \mathrm{kPa} \\ \text { Case (C) : } P_{p l}=-0.49 \sim-1.96 \mathrm{kPa}\end{array}\right)$

Frequency : $0.2 \mathrm{~Hz}$

\begin{tabular}{|c|c|c|c|c|c|c|}
\hline \multirow[b]{2}{*}{ Specimen } & \multicolumn{3}{|c|}{ Tidal volume $\bar{v}\left(\mathrm{~cm}^{3}\right)$} & \multicolumn{3}{|c|}{ Hysteresis area $\mathrm{H}(\boldsymbol{\mu} \mathrm{J})$} \\
\hline & Case ( $A)$ & Case (B) & Case (C) & Case $(A)$ & Case (B) & Case (C) \\
\hline $\begin{array}{l}I \\
I \\
I \\
V\end{array}$ & $\begin{array}{l}12.7 \\
12.4 \\
14.7 \\
18.5 \\
20.1\end{array}$ & $\begin{array}{l}20.3 \\
\text { 18. } 3 \\
22.3 \\
27.5 \\
29.3\end{array}$ & $\begin{array}{l}27.3 \\
23.6 \\
29.2 \\
34.9 \\
37.1\end{array}$ & $\begin{array}{l}0.41 \\
0.49 \\
0.48 \\
0.73 \\
1.28\end{array}$ & $\begin{array}{l}1.25 \\
1.20 \\
1.43 \\
1.87 \\
3.79\end{array}$ & $\begin{array}{l}\text { 2. } 64 \\
\text { 2. } 66 \\
\text { 2. } 91 \\
\text { 3. } 44 \\
6.62\end{array}$ \\
\hline
\end{tabular}

\begin{tabular}{|c|c|c|c|c|c|c|}
\hline \multicolumn{7}{|c|}{ Frequency : $5 \mathrm{~Hz}$} \\
\hline \multirow[b]{2}{*}{ Specimen } & \multicolumn{3}{|c|}{ Tidal volune $\bar{v}\left(\mathrm{~cm}^{3}\right)$} & \multicolumn{3}{|c|}{ Hysteresis area $\mathrm{H}(\mu \mathrm{J})$} \\
\hline & Case $(\Lambda)$ & Case (B) & Case $(\mathrm{C})$ & Case $(\Lambda)$ & Case (B) & Case $(C)$ \\
\hline $\begin{array}{l}\text { I } \\
\text { I } \\
\text { I } \\
\text { V }\end{array}$ & $\begin{array}{r}8.7 \\
8.2 \\
9.4 \\
10.0 \\
10.8\end{array}$ & $\begin{array}{l}13.9 \\
12.6 \\
14.4 \\
15.8 \\
16.1\end{array}$ & $\begin{array}{l}17.8 \\
16.1 \\
18.1 \\
19.6 \\
19.5\end{array}$ & $\begin{array}{l}2.03 \\
1.81 \\
2.31 \\
2.70 \\
3.28\end{array}$ & $\begin{array}{l}6.15 \\
4.77 \\
6.26 \\
7.82 \\
8.43\end{array}$ & $\begin{array}{c}11.8 \\
8.99 \\
11.6 \\
14.0 \\
14.2\end{array}$ \\
\hline
\end{tabular}

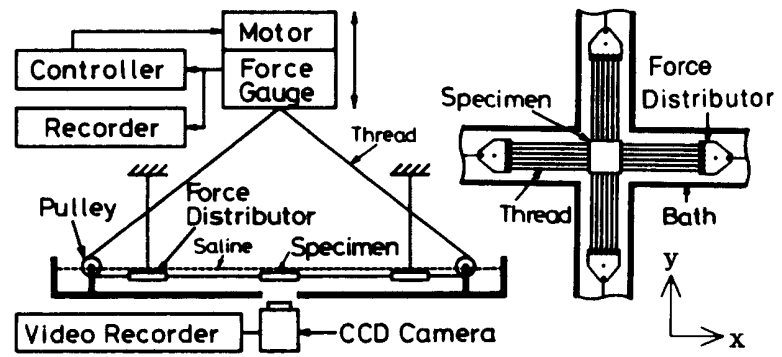

Fig. 3 Experimental apparatus for biaxial tension test of tissue specimen 
and the Lagrangian stress of the tissue are transformed from the deformation and the applied force, respectively.

The relationships between the stretch ratio and the Lagrangian stress in the $x$ direction are shown in Fig. 4( a ) for the tissue of the lung specimens I, III and $\mathrm{V}$. The result is almost identical to that in the $y$ direction. No significant difference is found between the loading and unloading curves, and the elastic properties of the tissue are determined on the basis of the loading curve in this study.

Tai and $L^{(22)}$ reported the homogeneity and isotropy of tissue elasticity over all regions of the lung parenchyma. Since uniform distribution of the stretch ratio is again experimentally confirmed in the target region, the tissue elasticity is described using the pseudoenergy function proposed by Fung ${ }^{(1)}$ under the assumption of uniform deformation and isotropy.

The Lagrangian stresses are calculated from the stretch ratio by the constitutive equation (15) in the Appendix under the plane stress $T_{z}=0$ condition. The stresses $T_{x m}^{(t)}$ and $T_{y m}^{(t)}(m=1,2, \cdots, M)$ denote the values calculated from the stretch ratios $\lambda_{x m}^{(e)}$ and $\lambda_{y m}^{(e)}$ in the $x$ and $y$ directions obtained by the experiment.

Table 2 Identified material constants for individual specimen

\begin{tabular}{c|c|c|c}
\hline Specimen & $c_{1}(\mathrm{~Pa})$ & $c_{2}$ & $c_{3}$ \\
\hline $\mathrm{I}$ & 19.1 & 1.48 & 0.117 \\
$\mathbf{I}$ & 22.1 & 1.75 & 0.411 \\
$\mathbf{I}$ & 39.7 & 1.90 & 0.509 \\
$\mathrm{~V}$ & 106 & 0.824 & 0.152 \\
\hline
\end{tabular}

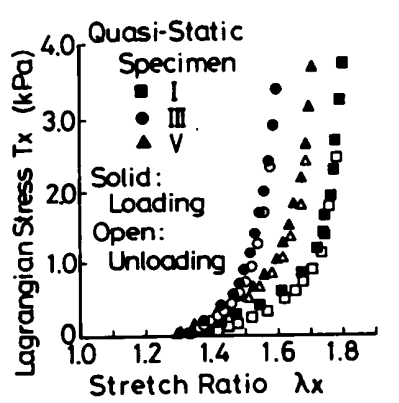

(a) At loading vs at unloading

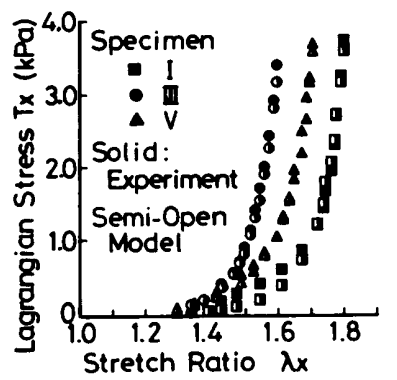

(b) Experimental vs theoretical

Fig. 4 Elastic properties of lung tissue
The material constants $c_{i}(i=1,2,3)$ in Eq. (13) are determined so as to minimize the sum of the square of the error

$$
R=\sum_{m=1}^{M}\left\{\left(T_{x m}^{(e)}-T_{x m}^{(t)}\right)^{2}+\left(T_{y m}^{(e)}-T_{y m}^{(t)}\right)^{2}\right\}
$$

where $T_{x m}^{(e)}$ and $T_{y m}^{(e)}$ are the experimental values of the Lagrangian stresses. The identified material constants are listed in Table 2 for individual lungs. Figure 4 ( b ) shows the experimental Lagrangian stresses and the theoretical values calculated from the material constants.

\section{Parameter Identification by Model-based Simulation}

This section discusses the indirect method of parameter identification. The identification process uses the experimental observations of the system level and the simulation model with parameters determined by the element-level experiment. The concept of personalized simulation is employed in this section.

\subsection{Scope and assumption of parameter identification}

Some parameters of the simulation model of lung respiration could not be directly determined through the element-level submodel and experiment. The indirect approach to parameter identification is a possibility which overcomes this difficulty. In this context, such model parameters could be determined on the basis of the system-level model and experiment. The lung volume in the natural state $V_{0}$, the hysteresis coefficient of the surface tension $a$ and the pressure loss coefficient $\zeta$ at branching points in the airway are the model parameters identified by the indirect method in this study. The following assumptions are imposed on the simulation model :

(a) As airway morphology of the rabbit lung, Weibel's data ${ }^{(4)}$ for the human lung are scaled down to one-third. The number of generations from mouth to alveoli is reduced to 17 .

(b) The upper bound and lower bound of surface tension are assumed to be $\gamma_{u}=30 \times 10^{-3} \mathrm{~N} / \mathrm{m}$ and $\gamma_{1}=$ $2 \times 10^{-3} \mathrm{~N} / \mathrm{m}$, respectively, with reference to previous reports $^{(23),(24)}$.

(c) The edge length of the cubic alveoli is $100 \mu \mathrm{m}$ in the idealized parenchymal model.

(d) Viscoelasticity of the lung tissue is ignored.

\section{2 Parameter identification procedure}

The pressure-volume change relation studied in section 3.1 is used as the experimental observation of the system level. The elastic properties in Table 2 identified in section 3.2 are used to simulate the dynamic behavior of the individual lung specimen. The boundary conditions are assumed to be identical to those of the experiment of the system level. That is, 
Table 3 Identified values of parameters $a$ and $V_{0}$ along with the errors in tidal volume and hysteresis area at quasi-static state (Frequency : $0.2 \mathrm{~Hz}$ )

\begin{tabular}{|c|c|c|c|c|c|c|c|}
\hline \multirow{2}{*}{ Specimen } & \multirow{2}{*}{$\zeta$} & \multicolumn{3}{|c|}{ Error of tidal volume $R_{\mathrm{v}, k}(\boldsymbol{x})$} & \multicolumn{3}{|c|}{ Error of hysteresis area $\mathbb{R}_{H_{1 k}}(\mathbf{x})$} \\
\hline & & Case $(\Lambda)$ & Case(B) & Case (C) & Case $(A)$ & Case (B) & $\operatorname{Case}(\mathrm{C})$ \\
\hline $\begin{array}{l}1 \\
1 \\
1 \\
y \\
y\end{array}$ & $\begin{array}{l}1.5 \\
3.0 \\
2.0 \\
1.5 \\
1.5 \\
\end{array}$ & $\begin{array}{r}2.3 \\
-14.9 \\
-9.5 \\
-3.7 \\
-10.4 \\
\end{array}$ & $\begin{array}{r}-6.3 \\
-18.7 \\
-14.2 \\
-8.9 \\
-10.0 \\
\end{array}$ & $\begin{array}{r}-10.7 \\
-22.1 \\
-16.9 \\
-9.9 \\
-36.3 \\
\end{array}$ & $\begin{array}{l}21.8 \\
16.3 \\
14.6 \\
12.8 \\
-4.7 \\
\end{array}$ & $\begin{array}{l}14.5 \\
24.9 \\
17.7 \\
13.9 \\
10.4 \\
\end{array}$ & $\begin{array}{r}2.8 \\
18.8 \\
9.8 \\
12.3 \\
46.0 \\
\end{array}$ \\
\hline
\end{tabular}

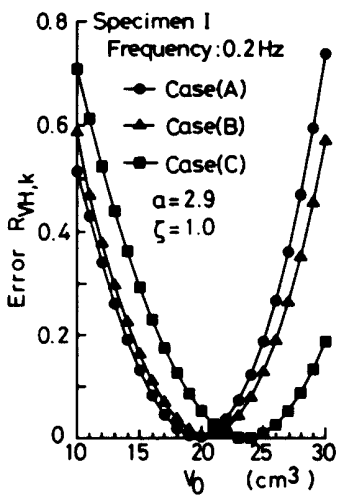

(a) With respect to lung volume $V_{0}$ at natural state

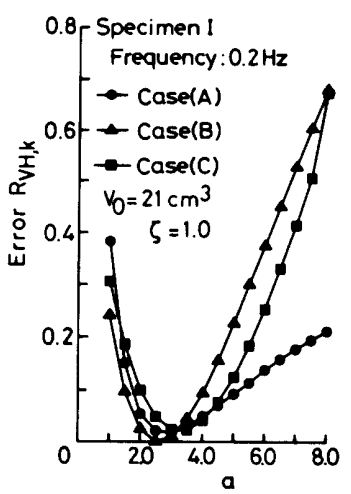

(b) With respect to hysteresis coefficient $a$

Fig. 5 Sensitivity of error in the vicinity of identified values (Lung specimen I)

the oral pressure is maintained at atmospheric pressure, and the pleural pressure is oscillated with the same frequency and amplitude as that used in the $P_{p l}$ $-\Delta V$ measurements. The criterion of minimum error is employed again in order to identify the model parameters mentioned in section 4.1. The error is defined by the characteristic values of $P_{p l}-\Delta V$ relations. That is, the tidal volume $\bar{V}_{k}^{(s)}$ and the hysteresis area $H_{k}{ }^{(s)}$ calculated from the simulated $P_{p l}-\Delta V$ relation take the place of the theoretical values, and the errors are defined as

$$
\begin{aligned}
& R_{V, k}=\left(\bar{V}_{k}^{(e)}-\bar{V}_{k}^{(s)}\right) / \bar{V}_{k}^{(e)} \\
& R_{H, k}=\left(H_{k}^{(e)}-H_{k}^{(s)}\right) / H_{k}^{(e)}
\end{aligned}
$$

between the simulated values and the experimental values $\bar{V}_{k}^{(e)}$ and $H_{k}^{(e)}$ listed in Table 1 , where the subscript $k$ distinguishes Cases (A), (B) and (C) with different amplitudes of pleural pressure. The model parameters $a, V_{0}$ and $\zeta$ are identified so as to minimize the resultant error

$$
\left.\begin{array}{l}
R_{V H}=\sum_{k} R_{V H, k} \\
R_{V H, k}=R_{V, k}^{2}+R_{H, k}^{2}
\end{array}\right\}
$$

over all cases.

\section{Identified Parameters and Personalized Simula- tion}

\subsection{Parameter identification at quasi-static state}

The coefficient of the surface tension $a$ and the lung volume in the natural state $V_{0}$ are identified from the $P_{p l}-\Delta V$ relations at the quasi-static state with the low frequency of $0.2 \mathrm{~Hz}$ of the pleural pressure oscillation. In this case of low frequency, the pressure loss coefficient $\zeta=\zeta_{i}$ is equal to 1.0 , because it is confirmed that the influence of the gas flow in the airway is negligible.

The values of the parameters are identified for individual lung specimens. The identified values are listed in Table 3 along with the errors in tidal volume and hysteresis area. The identified values of the parameters vary specimen by specimen. The errors in tidal volume and hysteresis area are kept at a reasonable level for every lung specimen and case, and the experimental situation is simulated under the identified values of the parameters in a quantitative manner.

The behavior of the case-wise resultant error $R_{V H, k}$ is examined against the value of the parameter $a$ or $V_{0}$ in the vicinity of the identified one (Fig. 5). No significant difference is found among the values of the parameters which minimize the error $R_{v H, k}$ for each case of amplitude of pleural pressure oscillation. This fact indicates that the identification process gives a meaningful result and that the identified values of the parameters $a$ and $V_{0}$ are commonly applicable to a certain range of amplitudes of pleural pressure oscillation, including those of Cases (A), (B) and (C). The simulated $P_{p t}-\Delta V$ relations with the identified values of the parameters are compared with the experimental results in Fig. 6 for specimen I. The simulated $P_{p l}$ $-\Delta V$ curves coincide reasonably with the experimental ones, where no smoothing treatment is applied.

In the case of low-frequency oscillation of pleural pressure, the hysteresis of the $P_{p l}-\Delta V$ relation is 
interpreted as the influence of surface tension at alveoli $^{(8),(10),(12)}$, although the dynamic behavior of the surface tension has not yet been measured directly in vivo. Figure 7 shows the relation between the surface tension and the area ratio indicated by the simulation using identified values of the parameters. The simple rate-type model of surface tension vs area ratio in Eq. (3) gives the exponent response as shown in Fig. 7. This may be the reason for the slight difference in $P_{\rho t}$ $-\Delta V$ relations between the simulation and the experiment, as is shown in Fig. 6. In spite of this difference in detail, the general agreement of the simulated and experimental $P_{p l}-\Delta V$ relations indicates the potential for predicting surface tension behavior inside of the lung by means of a simulation based on personalized model parameters.

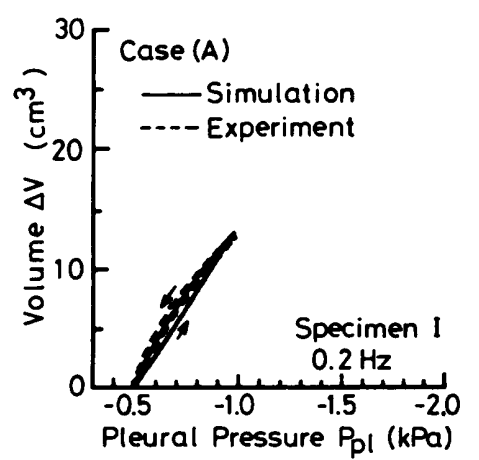

(a) Case (A) : $P_{p l}=-0.49 \sim-0.98 \mathrm{kPa}$

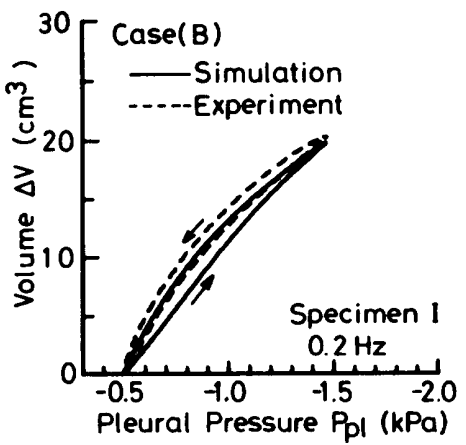

(b) Case(B) : $P_{p l}=-0.49 \sim-1.47 \mathrm{kPa}$

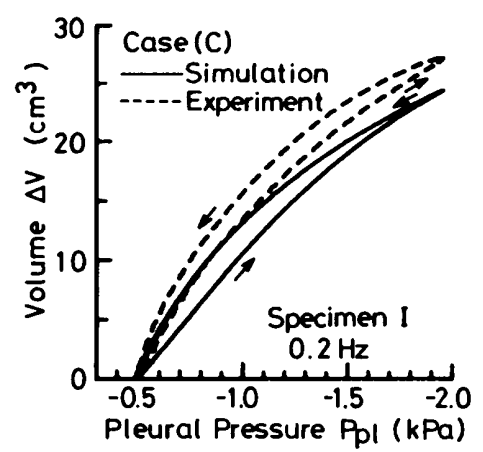

(c) $\operatorname{Case}$ (C) : $P_{p l}=-0.49 \sim-1.96 \mathrm{kPa}$

Fig. $6 P_{p l}-\Delta V$ relations in quasi-static state (Lung specimen I, $a=2.9, V_{D}=21 \mathrm{~cm}^{3}$ )

\subsection{Parameter identification at a dynamic state}

The pressure loss coefficient $\zeta$ at the branching points in the airway is identified from the $P_{p l}-\Delta V$ relation observed at the dynamic state with a frequency of pleural pressure oscillation of $5 \mathrm{~Hz}$. The parameters $a$ and $V_{0}$ in Table 2 are used as the known values.

Figure 8 represents the sensitivity of the casewise resultant error $R_{V H, k}$ with respect to the pressure loss coefficient in the vicinity of the identified values. The value of the pressure loss coefficient $\zeta$, which minimize the case-wise resultant error $R_{v H, k}$ are $\zeta=$ $2.5,1.5$, and 1.0 for Cases (A), (B) and (C), respectively. These values are not identical to each other, but the deviation is not significant. This supports the assumption that the pressure loss coefficient $\zeta$ is constant in the simulation of breathing dynamics under the moderate frequency condition, although the coefficient strictly depends on the gas velocity.

Table 4 gives the identified value of $\zeta$ which minimizes the resultant error $R_{V H}$ over the cases for individual lung specimens. The tidal volume and hysteresis area simulated under the identified values of $\zeta$ lead to the error for each lung specimen and case listed in this table. The simulated $P_{p l}-\Delta V$ relations

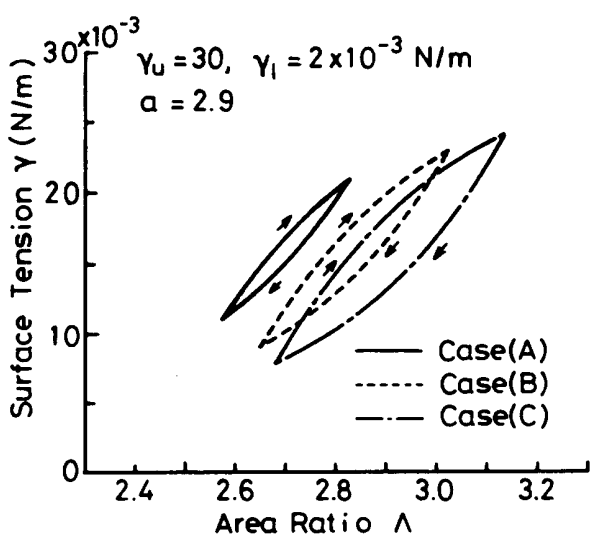

Fig. 7 Surface tension vs surface area ratio predicted by simulation

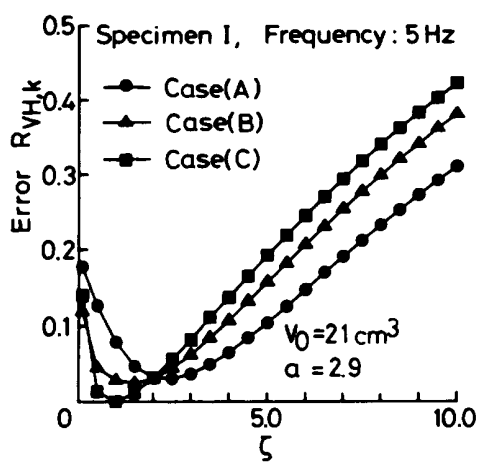

Fig. 8 Sensitivity of error with respect to pressure loss coefficient in the vicinity of the identified value (Lung specimen I) 
Table 4 Identified values of parameters $\zeta$ along with the errors in tidal volume and hysteresis area at dynamic state (Frequency : $5 \mathrm{~Hz}$ )

\begin{tabular}{|c|c|c|c|c|c|c|c|c|}
\hline \multirow[b]{2}{*}{ Specinen } & \multirow{2}{*}{$\begin{array}{c}V_{o} \\
\left(\mathrm{~cm}^{3}\right)\end{array}$} & \multirow[b]{2}{*}{ a } & \multicolumn{3}{|c|}{ Error of tidal volune $\mathbf{R}_{v_{k}}(\boldsymbol{x})$} & \multicolumn{3}{|c|}{ Error of hysteresis area $\mathbf{R}_{H, k}(\boldsymbol{x})$} \\
\hline & & & Case $(\AA)$ & Case(B) & Case(C) & Case $(\Lambda)$ & Case(B) & Case (C) \\
\hline $\begin{array}{l}1 \\
1 \\
\text { V }\end{array}$ & $\begin{array}{l}21 \\
22 \\
26 \\
18 \\
37\end{array}$ & $\begin{array}{l}2.9 \\
3.1 \\
2.7 \\
1.7 \\
3.9\end{array}$ & $\begin{array}{r}2.8 \\
-2.6 \\
2.8 \\
1.4 \\
-3.8\end{array}$ & $\begin{array}{r}-2.6 \\
-1.4 \\
-0.1 \\
0.1 \\
9.3\end{array}$ & $\begin{array}{r}-10.5 \\
-6.9 \\
-8.7 \\
-4.0 \\
9.0\end{array}$ & $\begin{array}{r}11.3 \\
18.5 \\
13.3 \\
3.4 \\
-13.1\end{array}$ & $\begin{array}{r}7.6 \\
6.4 \\
12.1 \\
5.4 \\
3.3\end{array}$ & $\begin{array}{r}-12.8 \\
-20.5 \\
-12.9 \\
-8.5 \\
3.2\end{array}$ \\
\hline
\end{tabular}

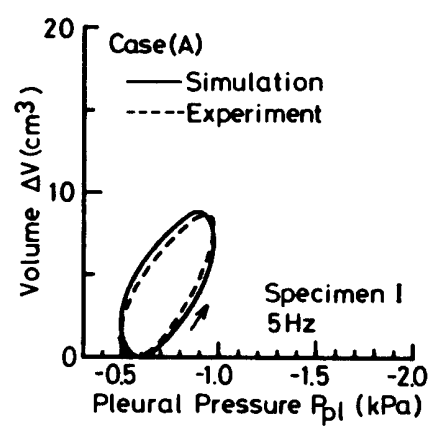

(a) Case (A): $P_{p l}=-0.49 \sim-0.98 \mathrm{kPa}$

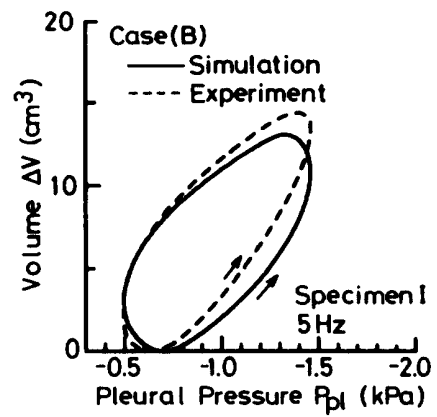

(b) $\operatorname{Case}$ (B) : $P_{p l}=-0.49 \sim-1.47 \mathrm{kPa}$

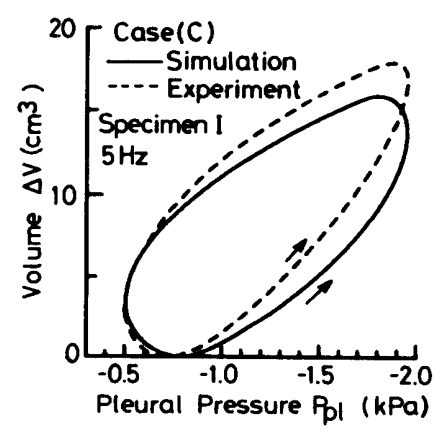

(c) $\operatorname{Case}(\mathrm{C}): P_{p l}=-0.49 \sim-1.96 \mathrm{kPa}$

Fig. $9 P_{p l}-\Delta V$ relation in dynamic state (Lung specimen I, $a=2.9, V_{0}=21 \mathrm{~cm}^{3}, \zeta=1.5$ )

are compared with the experimental ones in Fig. 9 for lung specimen I. Since the error listed in Table 4 includes that stemming from a priori identification of parameters $a$ and $V_{0}$, the error level in this table is slightly higher than that in Table 3 of the previous section. However, the error level is again maintained within a moderate range, and no significant difference is found between the simulated $P_{p l}-\Delta V$ relation and the experimental values, as seen in Fig. 9. The situation is very similar in the cases of other lung speci-

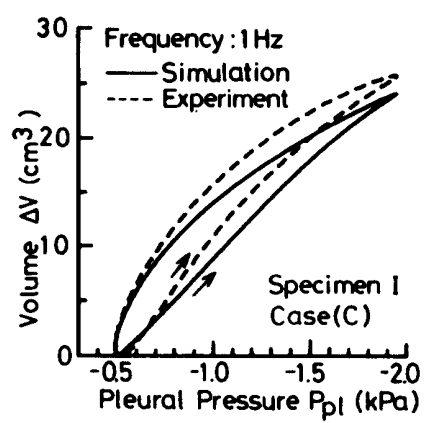

(a) $1 \mathrm{~Hz}$

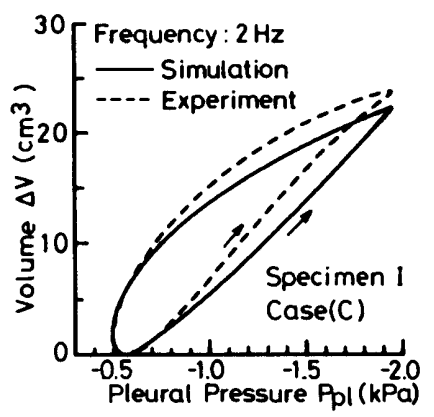

(b) $2 \mathrm{~Hz}$

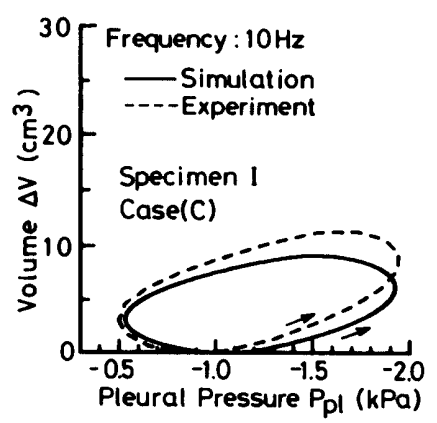

(c) $10 \mathrm{~Hz}$

Fig. $10 P_{p l}-\Delta V$ relation with several frequencies (Lung specimen I, $a=2.9, V_{0}=21 \mathrm{~cm}^{3}, \zeta=1.5$ )

mens. These results indicate that a simulation based on identified parameters for the individual lung has the potential capability to evaluate breathing dynamics quantitatively.

\section{3 Evaluation of simulation model}

In order to evaluate the simulation model with the model parameters identified for individual lung specimens, the dynamic response of the breathing is simulated for various conditions and compared with the experimental results.

The simulation is conducted for respiration under the frequencies of $1 \mathrm{~Hz}, 2 \mathrm{~Hz}$ and $10 \mathrm{~Hz}$. The parameters in Table 3 and Table 4 identified using the $P_{p l^{-}}$ $\Delta V$ relations at frequencies of $0.2 \mathrm{~Hz}$ and $5 \mathrm{~Hz}$ are used for the simulation. The simulated results for different frequencies show good agreement with the experimental values, as demonstrated in Fig. 10. This figure shows the case of the largest amplitude of pleural pressure oscillation, Case (C), which results 


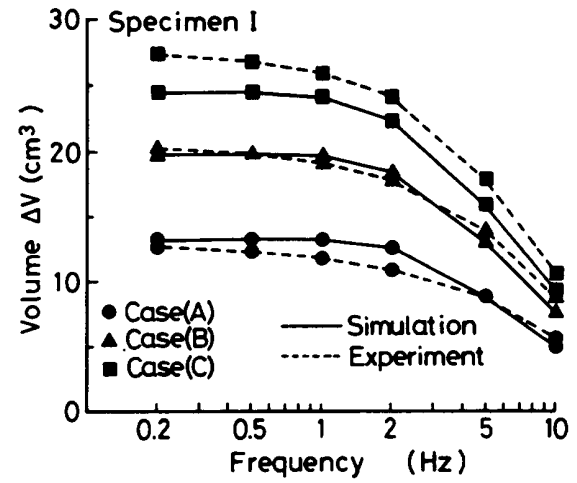

Fig. 11 Frequency response of tidal volume (Lung specimen I)

in the poorest agreement in Fig. 6 among the three cases. The degree of coincidence between the simulated and experimental results is quite similar to those of the other cases of amplitude of pleural pressure oscillation and to that of the remaining lung specimen. Figure 11 shows the frequency response diagram of the tidal volume plotted against the frequencies of $0.2 \mathrm{~Hz}$ to $10 \mathrm{~Hz}$ of pleural pressure oscillation in the case of lung specimen I. The simulated estimation agrees sufficiently with the experimental observation for Cases (A), (B) and (C). This result is representative of the capabilities of the simulation model to sufficiently investigate the system characteristics of breathing dynamics, such as the frequency response, and demonstrates that a simulation based on the identified model parameters is able to predict the behavior of the lung within the relatively wide range of breathing frequencies examined in this study. The personalization of the simulation model for breathing dynamics might be a promising approach to the quantitative investigation of lung respiration.

\section{Conclusions}

This study aimed to quantitatively identify the parameters of the simulation model for breathing dynamics of lung respiration. In the experimental part, the relationships between pleural pressure and volume change of the lung are observed in situ using excised rabbit lungs in their obtained state, and the elasticity of the tissue sliced from the lung is measured under biaxial tension. The simulation model consists of submodels of mechanical elements of breathing dynamics such as gas flow in the airway, tissue elasticity, surface tension activity in the alveoli and so on. The distributed parameter model is employed to the greatest possible extent. Some of the parameters required for the simulation model can be determined in the element-level submodel, and some cannot. Tissue elasticity is a typical instance of the former category, and it is directly determined in a tissue-level model. The lung volume in the natural state, the hysteresis coefficient of the surface tension and the pressure loss coefficient at the airway branches belong to the latter category, and they were identified in an indirect manner where by the simulation result is combined with an experimental observation at the system level. Personalization is incorporated into the system model in order to further quantify the simulation.

The $P_{p l}-\Delta V$ relations simulated under identified values of the model parameters sufficiently coincide with the experimental results observed at various amplitudes of pleural pressure oscillation, and it was confirmed that a combination of the identified values of the parameters has the capability to quantitatively predict breathing dynamics for various conditions by means of simulation. This indicates that the established simulation model represents substantial structure and mechanisms of the mechanical aspect of breathing dynamics.

The concept of analysis by synthesis in the simulation procedure allows investigation of a complex system such as lung respiration. A simulation based on personal properties has high potential not only to estimate phenomena which could not be examined experimentally in vivo, but also to cope with the differences between individuals. Such a personalized insight to a living system is the key to the evolution of biomechanical consideration in all medical fields.

\section{Appendix}

\section{Description of lung elasticity}

A pseudostrain energy function of the lung parenchyma

$$
W=\frac{1}{2} c_{1} \exp \left\{c_{2}\left(E_{x}^{2}+E_{y}^{2}\right)+2 c_{3} E_{x} E_{y}\right\}
$$

+Symmetrical terms by permutation

has been proposed by Fung ${ }^{(1)}$, where $c_{j}(j=1,2,3)$ are material constants and $E_{i}(i=x, y, z)$ stand for Green's strains defined as

$$
E_{i}=\frac{1}{2}\left(\lambda_{i}^{2}-1\right)
$$

using the stretch ratio $\lambda$ of the lung tissue. Lagrangian stresses $T_{i}(i=x, y, z)$ are derived as the derivative of the pseudostrain energy function with respect to the stretch ratio, and the constitutive equation is written as

$$
T_{i}=\frac{\partial W}{\partial \lambda_{i}}
$$

For example, the $x$ directional component becomes

$$
\begin{aligned}
T_{x} & =c_{1} \lambda_{x}\left[\left(c_{2} E_{x}+c_{3} E_{y}\right)\right. \\
& \times \exp \left\{c_{2}\left(E_{x}^{2}+E_{y}^{2}\right)+2 c_{3} E_{x} E_{y}\right\} \\
& +\left(c_{2} E_{x}+c_{3} E_{z}\right) \\
& \left.\times \exp \left\{c_{2}\left(E_{x}^{2}+E_{z}^{2}\right)+2 c_{3} E_{x} E_{z}\right\}\right] .
\end{aligned}
$$


The idealized parenchyma model composed of cubic alveoli with edge length $\Delta$ yields isotropic and uniform deformation of the lung expressed by

$$
\begin{aligned}
& T_{x}=T_{y}=T_{z}=T, \\
& \lambda_{x}=\lambda_{y}=\lambda_{z}=\lambda, \\
& E_{x}=E_{y}=E_{z}=E .
\end{aligned}
$$

Thus, the macroscopic stress $\sigma_{e}$ due to tissue elasticity is given as

$$
\sigma_{e}=\frac{2 c_{1}}{\lambda}\left(c_{2}+c_{3}\right) E \exp \left\{2 E^{2}\left(c_{2}+c_{3}\right)\right\}
$$

\section{References}

(1) Fung, Y. C., Stress, Deformation, and Atelectasis of the Lung, Circul. Res., Vol. 37 (1975), p. 481.

( 2 ) Vawter, D. L., Fung, Y. C. and West, J. B., Constitutive Equation of Lung Tissue Elasticity, Trans. ASME, J. Biomech. Eng., Vol. 101, No. 1 (1979), p. 38.

(3) Lanir, Y., Constitutive Equations for the Lung Tissue, Trans. ASME, J. Biomech. Eng., Vol. 105 (1983), p. 374.

(4) Weibel, E. R., Morphometry of the Human Lung, (1963), Academic Press, New York.

( 5 ) Horsfield, K. and Cumming, G., Morphology of Branching Tree in Man, J. Appl. Physiol., Vol. 24, No. 3 (1968), p. 373.

(6) Pedley, T. J., Schroter, R. C. and Sudlow, M. F., Flow and Pressure Drop in Systems of Repeatedly Branching Tubes, J. Fluid Mech., Vol. 46, Part 2 (1971), p. 365

(7) Hoshimiya, N., Tanaka, M. and Matsuo, M., Finite-length collapsible-tube model of the check -valve in expiration, Japanese J. Medical Elec. tronics and Biological Eng. (in Japanese), Vol. 6, No. 6 (1974), p. 181.

(8) Clements, J. A. and Tierney, D. F., Alveolar Instability Associated with Altered Surface Tension, Handbook Physiology (Eds. Fenn, W. O. and Rahn, H.), Respiration, Vol. 2, Sec. 3 (1965), p. 1565, Am. Physiol. Soc., Washington, D.C.

(9) Wichert, P. von and Muller, B., Basic Research of Lung Surfactant, Progress in Respiration Research (Herzog, H. ed.), Vol. 25 (1990), Karger, Basel.

(10) Stamenovic, D. and Wilson, T. A., A Strain Energy Function for Lung Parenchyma, Trans. ASME, J. Biomech. Eng., Vol. 107 (1985), p. 81.

(11) Kimmel, E. and Budiansky, B., Surface Tension and the Dodecahedron Model for Lung Elasticity, Trans. ASME, J. Biomech. Eng., Vol. 112, No. 2 (1990), p. 160.

(12) Bachofen, H., Hildebrandt, J. and Bachofen, F., Pressure-Volume Curves of Air- and Liquid-filled Excised Lung (Surface Tension in Situ, J. Appl.
Physiol., Vol.29, No. 4 (1970), p. 442.

(13) Hajji, M. A., Wilson, T. A. and Lai-Fook, S. J., Improved Measurements of Shear Modulus and Pleural Membrane Tension of the Lung, J. Appl. Physiol., :Respiratory and Environmental Exercise Physiology, Vol. 47, No. 1 (1979), p. 175.

(14) Seguchi, Y., Fung, Y. C. and Maki, H., Computer Simulation of Dynamics of Fluid-Tissue Systems due to Discretization Procedure and its Application to Respiratory Dynamics, Proc. 1st China, Japan \& U.S.A. Conf. Biomech., (Eds. Fung, Y. C., Fukuda, E. and Wang, J. J.) (1984), p. 224, Science Press.

(15) Seguchi, Y., Fung, Y. C. and Ishida, T., Respiratory Dynamics - Computer Simulation, Frontier in Biomech., (Eds. Schmid-Schonbein, G. W., Woo, S. L-Y and Zweifach, B. W.) (1985), p. 377, Springer-Verlag.

(16) Seguchi, Y., Fung, Y. C. and Nagatani, H., Respiratory Dynamics-Surface Tension Influence, Proc. 8th Annual Conf. IEEE/Eng. Medicine \& Biology, 8th Annl. Conf., Vol. 2(1986), p. 889, Ft. Worth.

(17) Wada, S., Seguchi, M. and Tanaka, M., A Simulation of Respiratory Dynamics and the Surface Tension Influence, Trans. Jpn. Soc. Mech. Eng., (in Japanese), Vol. 54, No. 505, C (1988), p. 2069.

(18) Wada, S., Seguchi, M. and Tanaka, M., Breathing -Ventilation Model, and Simulation of High-Frequency Ventilation, Trans. Jpn. Soc. Mech. Eng., (in Japanese), Vol. 56, No. 525, A (1990), p. 1303.

(19) Wada, S., Seguchi, Y. and Tanaka, M., Simulation of Breathing and Ventilation Combined with Body Circulation, 1990 Advances in Bioengineering (Ed. Goldstein, S. A.), 1990, p. 67, ASME, New York.

(20) Seguchi, Y., Wada, S., Tanaka, M. and Togari, H., Model, Simulation and Applications on Total Respiration in terms of Computational Biomechanics, Computational Mechanics ' 91 (Eds. At luri, S. N., Beskos, D. E., Jones, R. and Yagawa, G.), (1991), p. 924, ICES Publications, Atlanta.

(21) Vawter, D. L., Fung, Y. C. and West, J. B., Elasticity of Excised Dog Lung Parenchyma, J. Appl. Physiol., :Respiratory and Environmental Exercise Physiology, Vol. 45, No. 2 (1978), p. 261.

(22) Tai, R. C. and Lee, G. C., Isotropy and Homogeneity of Lung Tissue Deformation, J. Biomech., Vol. 14, No. 4 (1981), p. 243.

(23) Smith, J. C. and Stamenovic, D., Surface forces in Lungs (Alveolar Surface Tension-Lung Volume Relationships), J. Appl. Physiol., Vol.60, No. 4 (1986), p. 1341.

(24) Schürch, S., Surface Tension at Low Lung Volumes: Dependence on Time and Alveolar Size, Respiration Physiol., Vol. 48 (1982), p. 339. 